\title{
CONSENSUS PROBLEMS WITH DISTRIBUTED DELAYS, WITH APPLICATION TO TRAFFIC FLOW MODELS
}

\author{
WIM MICHIELS*, CONSTANTIN-IRINEL MORĂRESCU †, AND SILVIU-IULIAN \\ NICULESCU $\ddagger$
}

\begin{abstract}
This paper focuses on consensus problems for a class of linear systems with distributed delay that are encountered in modeling traffic flow dynamics. In the application problems the distributed delay, whose kernel is a gamma-distribution with a gap, represents the human drivers' behavior in the average. The aim of the paper is to give a characterization of the regions in the corresponding delay parameter space, where a consensus is reached for all initial conditions. The structure and properties of the system are fully exploited, which leads to explicit and computationally tractable expressions. As a by-product a stability theory for distributed delay systems with a gamma-distribution kernel is developed. Also explicit expressions for the consensus function(al) of time-delay systems with constant and distributed delays that solve a consensus problem are provided. Several illustrative examples complete the presentation.
\end{abstract}

Keywords: consensus problem, time-delays, traffic model, stability.

1. Introduction. Networks of agents are typically large-scale interconnected systems whose dynamics depend on the topology of the network but also on the individual behaviors of the agents. In this context, agreements and cooperation between agents are needed in order to achieve some common, global objective. Roughly speaking, in a general setting the realization of a consensus consists of finding rules and strategies for reaching an agreement regarding some certain quantity of interest depending on the states of all the agents (see, e.g., [20, 23] for a recent survey on the topic).

Consider now a consensus protocol for a multi-agent system with a fixed, directed network topology and a distributed delay in the communication channels (see, e.g., $[19,13]$ and the references therein). More precisely, let the directed graph

$$
\mathcal{G}(V, E, \mathbb{A})
$$

be characterized by the node set $V=\{1, \ldots, p\}$, a set of edges $E$ where $(k, l) \in E$ if and only if $\alpha_{k, l} \neq 0$, and a weighted adjacency matrix $\mathbb{A}$ with zero diagonal entries and non-diagonal entries equal to $\alpha_{k, l}$. Let each node correspond to an agent whose dynamics are described by

$$
\dot{v}_{k}(t)=u_{k}(t), k=1, \ldots p .
$$

Furthermore, consider the following protocol:

$$
u_{k}(t)=\sum_{(k, l) \in E} \alpha_{k, l} \int_{0}^{\infty} f(\theta)\left(v_{l}(t-\theta)-v_{k}(t-\theta)\right) \mathrm{d} \theta, \quad k=1, \ldots, p,
$$

where $f(\cdot)$ denotes some delay kernel, and the notation $\lfloor\cdot\rfloor$ stands for

$$
\lfloor l\rfloor= \begin{cases}l, & l=1, \ldots, p, \\ \lfloor l+p\rfloor, & l<1 .\end{cases}
$$

\footnotetext{
*Department of Computer Science, Katholieke Universiteit Leuven, Celestijnenlaan 200A, 3001 Heverlee, Belgium, tel.+32.16.327537, fax +32.16.327996 (Wim.Michiels@cs.kuleuven.be).

†INRIA Rhône Alpes, BIPOP project, Inovalée, 655 avenue de l'Europe, 38330, Montbonnot, France (constantin.morarescu@inrialpes.fr) and University "Politehnica" of Bucharest, Department of Mathematics, Bucharest, Romania.

¥Laboratoire de Signaux et Systèmes (L2S, UMR CNRS 8506), CNRS-Supélec, 3, rue Joliot Curie, 91192, Gif-sur-Yvette, France (Silviu.Niculescu@hds.utc.fr).
} 
We assume that

$$
\alpha_{k, l} \geq 0, \quad k=1, \ldots, p, l=1, \ldots, p, k \neq l
$$

and that the graph $\mathcal{G}$ is strongly connected (see, e.g. [19] for the definition).

Let us discuss some of the motivations for introducing such a model. It is wellknown and well-accepted that networks and, more general, interconnected systems are subject to propagation and communication delays. If such delays are not critical in the perception and the observation of various behaviors, they may become critical if they are used in decision-making, control or consensus problems. The proposed model enters in this category.

In this context, most of the cases treated and presented in the literature consider only constant (piece-wise) delays. If such an assumption can be seen as sufficient for some examples, it becomes quite restrictive and conservative for others and among the applications concerned by such an argument, we can cite traffic dynamics. Note that the corresponding models are inherently time-delayed because of the limited sensing and acting capabilities of drivers against velocity and position variations [8, 12].

The idea of using delays in traffic flow dynamics is not new, and, to the best of the authors' knowledge was pointed out in the 60s (see, for instance, [2]). According to its origin (see, e.g. [8]), we can classify the delays in the traffic flow dynamics as follows: physiological delays (mainly induced by the human operators), mechanical time delays (time needed for the vehicle's response after some driver's action) and delays in the vehicles' action, to cite only a few (see also [25]).

Without any deep discussions on the modeling of the traffic dynamics, one of the simplest model often discussed in the literature is the (microscopic) car-following model, describing the behavior of multiple vehicles under the influence of a single constant time-delay $[2,12,24]$. In general, two spatial configurations are dealt with: the linear and the ring configuration. In what follows, for the sake of brevity, we shall only consider the ring configuration when discussing the traffic flow application, but the obtained results can also be applied to the linear configuration (see, e.g. [27] for further discussions concerning these configurations). The linear model of [2] can be written conceptually as follows:

$$
\dot{v}_{k}(t)=\alpha_{k}\left(v_{k-1}(t-\tau)-v_{k}(t-\tau)\right), k=1, \ldots, p,
$$

where $p$ is the number of considered vehicles and $v_{0}=v_{p}$. The left hand side represents the acceleration of the $k^{t h}$ vehicle, and the right hand side expresses the velocity difference of consecutive vehicles (see also [24] for a multiple cars model). One of the limitations of the model above is that, in general, humans retain a short-term memory of the past events and this may affect their control decision strategy and such a behavior cannot be described by using point-wise (or discrete) delays in the model. Furthermore, the drivers' perception and interpretations of the stimuli depend on various parameters, and are different from one driver to another. As pointed out in [27], a more realistic model should include a delay distribution over the time that depicts the human behavior in average. Conceptually, defining the delay distribution represents a challenging problem itself, and is far from being solved. In [27], the authors proposed three types of delay distributions: a uniform distribution, a $\gamma$-distribution and a $\gamma$-distribution with a gap, where the gap corresponds to the minimum reaction time of the humans with respect to some external signals and/or stimuli. In this paper we shall assume the third type of distribution. Remarks and 
discussions on its applications to other problems from engineering and biology can be found in [16]. Finally, it is important to point out that distributed delays are often encountered in controlling time-delay systems. Typical examples are given by the finite-spectrum assignment problems or the approximation of the derivative actions by its delay-difference counterpart (see, e.g. $[9,15]$ ).

The above discussions lead us to the analysis of the model

$$
\dot{v}_{k}(t)=\sum_{i=1}^{p-1} \alpha_{k,\lfloor k-i\rfloor} \int_{0}^{\infty} f(\theta)\left(v_{\lfloor k-i\rfloor}(t-\theta)-v_{k}(t-\theta)\right) \mathrm{d} \theta, \quad k=1, \ldots, p,
$$

where, as mentioned above, $f(\cdot)$ denotes the delay kernel. It is easy to see that the model (1.6) is nothing else than the protocol (1.3) applied to the agents described by (1.2). Since the delay distribution is assumed to be a gamma-distribution with a gap, the kernel $f$ is given by

$$
f(\xi)= \begin{cases}0, & \xi<\tau, \\ \frac{(\xi-\tau)^{n-1} \mathrm{e}^{-\frac{\xi-\tau}{T}}}{T^{n}(n-1) !}, & \xi \geq \tau,\end{cases}
$$

where $n \in \mathbb{N}, T>0$ and $\tau \geq 0$. Note that $f(\xi) \geq 0$ for all $\xi \geq 0$ and $\int_{0}^{\infty} f(\xi) d \xi=1$. The gap is defined by $\tau$, and the corresponding average delay of (1.7) satisfies:

$$
\tau_{m}=\int_{0}^{\infty} \xi f(\xi) d \xi=\tau+n T .
$$

As $T \rightarrow 0+$, the kernel (1.7) tends to a Dirac impulse centered at $\xi=\tau$ and (1.6) therefore reduces to a system with a point-wise delay $\tau$. As we shall see, the transition to $T=0$ is smooth from a stability point of view, as the stability determining eigenvalues are continuous w.r.t. $T \geq 0$.

The aim of the paper is to analyze the general consensus problem (1.2)-(1.3) for a particular delay kernel ( $\gamma$-distribution with a gap), more precisely, to perform a stability analysis of (1.6)-(1.7) with respect to the parameters $(T, \tau)$ and $n$, which determine the shape of the delay distribution. For a given value of $n$ we will determine regions in the $(T, \tau)$ space, such that for all initial conditions a consensus is reached. In the traffic flow application this corresponds to the fact that all cars eventually get the same speed. Note that the corresponding problem for an undirected graph (where $\mathbb{A}$ is symmetric) and a constant time-delay was investigated in [19].

The structure of the paper is as follows: in Section 2 the stability theory for systems with distributed delay is addressed, with the emphasis on consensus problems. Section 3 is devoted to the derivation of conditions on the pair $(T, \tau)$ for which (1.6)(1.7) realizes a consensus. Illustrative examples are given in Section 4. The analysis of other types of delay models for modeling traffic flow dynamics, in particular the socalled optimal velocity models, is briefly commented on in Section 5. Some concluding remarks are formulated in Section 6.

The following standard notation will be adopted: $\mathbb{C}\left(\mathbb{C}^{+}, \mathbb{C}^{-}\right)$is the set of complex numbers (with strictly positive, and strictly negative real parts), and $j=\sqrt{-1}$. For $z \in \mathbb{C}, \angle(z) \in(-\pi \pi], \Re(z)$ and $\Im(z)$ define the argument, the real part and the imaginary part of $z . \mathbb{R}\left(\mathbb{R}^{+}, \mathbb{R}^{-}\right)$denotes the set of real numbers (larger or equal to zero, smaller or equal to zero). $\mathbb{N}$ is the set of natural numbers, including zero and $\mathbb{Z}$ 
the set of integers. The set $\mathcal{C}\left(\mathcal{I}, \mathbb{C}^{p}\right)$, with $\mathcal{I} \subseteq \mathbb{R} \cup\{ \pm \infty\}$ and $p \in \mathbb{N}$ is the space of continuous functions from $\mathcal{I}$ to $\mathbb{C}^{p}$. Finally, the following functions will be used:

Definition 1.1. For $n \in \mathbb{N}$, let $g_{n}: \mathbb{R}^{+} \rightarrow \mathbb{R}^{+}$be such that $y=g_{n}(x)$ is the positive solution of $\left|y(1+j y)^{n}\right|=x$.

2. Stability theory for systems with distributed delays. Motivated by the structure of (1.6)-(1.7) we develop a stability theory for systems with an unbounded distributed delay of the form

$$
\dot{x}(t)=A \int_{0}^{\infty} f(\theta) x(t-\theta) d \theta,
$$

where $x(t) \in \mathbb{C}^{p \times 1}, A \in \mathbb{C}^{p \times p}$ and $f$ is given by (1.7). The approach is based on establishing relations with stability properties of general systems with bounded delay of the form

$$
\dot{x}(t)=\int_{-\tau}^{0} d \eta(\theta) x(t+\theta), x(t) \in \mathbb{C}^{r \times r},
$$

where $\eta(\theta), \theta \in[-\tau, 0]$ is a $\mathrm{r}$-by-r matrix whose elements are of bounded variation, because for such systems a well established stability theory exists [10].

A solution of $(2.1)$ is uniquely determined for an initial condition $\phi$, which belongs to the set $\mathcal{F}\left((-\infty, 0], \mathbb{C}^{p \times 1}\right)$, defined as

$\mathcal{F}\left((-\infty, 0], \mathbb{C}^{p \times 1}\right):=\left\{\phi \in \mathcal{C}\left((-\infty, 0], \mathbb{C}^{p \times 1}\right):\|\phi\|_{f}:=\int_{-\infty}^{0}\|f(-\theta) \phi(\theta)\|_{2} d \theta<\infty\right\}$

and equipped with $\|\cdot\|_{f}$. Denote by $t \in(-\infty, \infty) \rightarrow x(\phi)(t)$ the forward solution of (2.1) with initial condition $\phi$. In this way, stability definitions can be formulated in a similar way as in for systems with constant delays, see e.g. $[10,15]$ for the latter. We say, for instance, that the zero solution of (2.1) is asymptotically stable if and only if

$$
\begin{aligned}
& \forall \epsilon>0 \exists \delta>0 \forall \phi \in \mathcal{F}\left((-\infty, 0], \mathbb{C}^{p \times 1}\right)\|\phi\|_{f}<\delta \Rightarrow \forall t \geq 0\|x(\phi)(t)\|_{2}<\epsilon, \\
& \forall \phi \in \mathcal{F}\left((-\infty, 0], \mathbb{C}^{p \times 1}\right) \lim _{t \rightarrow \infty} x(\phi)(t)=0 .
\end{aligned}
$$

The substitution of a sample solution of the form $x(t)=e^{s t} X$, with $X \in \mathbb{C}^{p \times 1}$, in (2.1) leads us to the characteristic equation

$$
\operatorname{det}(s I-A F(s))=0
$$

where $F(s)$ is the Laplace transform of $f$. When $f$ is given by (1.7) the characteristic equation above rewrites as:

$$
\operatorname{det}\left(s I-A \frac{e^{-s \tau}}{(1+s T)^{n}}\right)=0
$$

which can be factored as

$$
\Pi_{k=1}^{p}\left(s-\frac{\mu_{k} e^{-s \tau}}{(1+s T)^{n}}\right)=0
$$

with $\mu_{k}, k=1, \ldots, p$, the eigenvalues of $A$. As we shall see, the roots distribution of (2.3)-(2.4) determines the stability properties of (2.1). However, the commonly 
used arguments, which are based on a spectral decomposition of the solutions (see, for instance, $[6,10]$ ) cannot be directly applied to a system of the form (2.1). A major obstacle is the fact that functions of the form $e^{s t} X, t \leq 0$, do not belong to the space $\mathcal{F}\left((-\infty, 0], \mathbb{C}^{p \times 1}\right)$ if $\Re(s)<1 / T$. We shall therefore develop arguments based on a comparison system.

Formally, with

$$
y(t)=\int_{0}^{\infty} f(\theta+\tau) x(t-\theta) d \theta=\int_{-\infty}^{t} f(t+\tau-\theta) x(\theta) d \theta,
$$

we get

$$
\left\{\begin{aligned}
y^{\prime}(t) & =\int_{-\infty}^{t} f^{\prime}(t+\tau-\theta) x(\theta) d \theta \\
& \vdots \\
y^{(n-1)} & =\int_{-\infty}^{t} f^{(n-1)}(t+\tau-\theta) x(\theta) d \theta \\
y^{(n)}(t) & =f^{(n-1)}(\tau) x(t)+\int_{-\infty}^{t} f^{(n)}(t+\tau-\theta) x(\theta) d \theta
\end{aligned}\right.
$$

which leads to

$$
\begin{aligned}
\left(T \frac{d}{d t}+I\right)^{n} y(t) & =T^{n} f^{(n-1)}(\tau) x(t)+\int_{-\infty}^{t}\left(T \frac{d}{d t}+I\right)^{n} f(t+\tau-\theta) d \theta \\
& =x(t)
\end{aligned}
$$

We conclude that a solution $x(\phi)(t)$ of $(2.1)$ satisfies

$$
\left\{\begin{array}{ccc}
\dot{x}(t) & = & A y(t-\tau) \\
\left(T \frac{d}{d t}+I\right)^{n} y(t) & = & x(t)
\end{array}\right.
$$

for $t \geq 0$, if (2.5), interpreted as an initial value problem, is accordingly initialized with

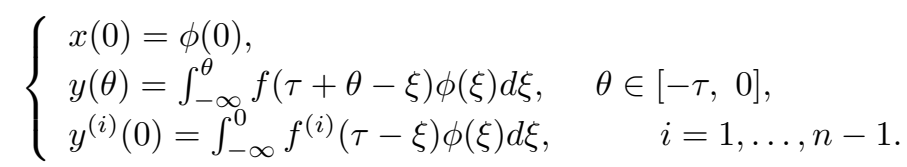

Note that the integrals in the right hand side of (2.6) are defined and bounded because $f^{(i)}(\xi), i=1, \ldots, n-1$ has the same asymptotic behavior as $f(\xi)$ as $\xi \rightarrow \infty$.

When letting $z=\left[\begin{array}{lll}x^{T} & \left.y^{T} y^{T} \cdots\left(y^{(n-1}\right)\right)^{T}\end{array}\right]^{T}$, the comparison system (2.5) can be written in a first order form as

$$
\dot{z}(t)=\bar{A} z(t)+\bar{B} z(t-\tau),
$$

where

$$
\bar{A}=\left[\begin{array}{ccccc}
0 & 0 & 0 & \cdots & 0 \\
0 & 0 & I & & \\
& & & \ddots & \\
\frac{I}{T^{n}} & -\left(\frac{n}{n}\right) \frac{1}{T^{n}} & & \cdots & -\left(\frac{n}{1}\right) \frac{1}{T^{1}}
\end{array}\right] \text { and } \bar{B}=\left[\begin{array}{ccccc}
0 & A & 0 & \cdots & 0 \\
0 & 0 & 0 & & 0 \\
\vdots & & & & \vdots \\
0 & & \cdots & & 0
\end{array}\right]
$$

The initial conditions for $(2.7)$ are assumed to belong to the space $\mathcal{C}\left([-\tau, 0], \mathbb{C}^{(n+1) p \times 1}\right)$. The next lemma summarizes the established relation between the solutions of (2.1) and (2.7) and also contains a partial converse: 
LEMma 2.1. If $x(t), t \in \mathbb{R}$, is a solution of (2.1) then there exists a solution $z(t), t \geq-\tau$ of (2.7) such that $[I 0 \cdots 0] z(t)=x(t)$ for all $t \geq-\tau$. If (2.7) has a solution of the form $Z e^{s t}, t \geq-\tau$, where $\Re(s) \geq 0$ and $Z \in \bar{C}^{(n+1) p \times 1} \backslash\{0\}$, then $\left[\begin{array}{lll}I & 0 & \cdots\end{array}\right] Z e^{s t}, t \in \mathbb{R}$, is a non-trivial solution of (2.1).

Proof. The first assertion follows from the above construction, and an extension of $(2.6)$ on the interval $[-\tau, 0]$.

To prove the second assertion, we partition $Z$ according to the structure of $\bar{A}$ and $\bar{B}$ as $Z=\left[\begin{array}{lll}X^{T} & Y_{0}^{T} \cdots Y_{n-1}^{T}\end{array}\right]^{T}$. Substituting $Z e^{s t}$ in (2.7) yields

$$
\begin{array}{r}
\left(s I-A \frac{e^{-s \tau}}{(1+s T)^{n}}\right) X=0, \\
Y_{i}=\frac{s^{i}}{(1+s T)^{n}} X, \quad i=0, \ldots, n-1 .
\end{array}
$$

It follows that $Z \neq 0$ if and only if $X \neq 0$. Furthermore, (2.8) implies that $X e^{s t}$ satisfies (2.1) for all $t \in \mathbb{R}$. The function $X e^{s t}, t \in \mathbb{R}$, is a solution since $\Re(s) \geq 0$ and thus $X e^{s t}, t \leq 0$, belongs to $\mathcal{F}\left((-\infty, 0], \mathbb{C}^{p \times 1}\right)$.

The system (2.7) is of the form (2.2) and corresponds to taking

$$
\eta(\theta)= \begin{cases}-\bar{A}-\bar{B}, & \theta=-\tau \\ -\bar{A}, & \theta \in(-\tau, 0) \\ 0, & \theta=0\end{cases}
$$

¿From $[10,6]$ the zero solution of (2.2)is asymptotically stable if and only if all the roots of its characteristic equation,

$$
\operatorname{det}\left(s I-\int_{-\tau}^{0} d \eta(\theta) e^{s \theta}\right)=0
$$

are in $\mathbb{C}^{-}$. For $(2.7)$ the characteristic equation reduces to:

$$
\operatorname{det}\left(s I-\bar{A}-\bar{B} e^{-s \tau}\right)=0,
$$

or, equivalently,

$$
\operatorname{det}\left(\left[\begin{array}{cc}
s I & -A e^{-s \tau} \\
-I & (1+s T)^{n}
\end{array}\right]\right)=0,
$$

The roots of (2.11) are called eigenvalues of (2.5) in what follows.

Note that (2.11) reduces to $(2.3)$ if $s \neq-1 / T$. Combining this result with Lemma 2.1 results in:

Proposition 2.2. The zero solution of (2.1) is asymptotically stable if and only if all roots of (2.3) are in $\mathbb{C}^{-}$.

Next, we derive conditions on the characteristic roots for which the linear system with unbounded distributed delay (2.1) solves a consensus problem. This stability property is defined in the following way:

DEFINITION 2.3. The system (2.1) solves a consensus problem if and only if

$$
\forall \phi \in \mathcal{F}\left((-\infty, 0], \mathbb{C}^{p \times 1}\right) \lim _{t \rightarrow \infty} x(\phi)(t)=\chi(\phi) E_{0},
$$

where $\chi(\phi) \in \mathbb{C}$ and $E_{0}=[1 \cdots 1]^{T}$. The function $\chi: \mathcal{F}\left((-\infty, 0], \mathbb{C}^{p \times 1}\right) \rightarrow \mathbb{C}$ is called the consensus functional. The system (2.1) solves a nontrivial consensus 
problem if and only if it solves a consensus problem and the consensus functional is not identically zero.

We follow the same methodology as for the asymptotic stability condition: we first address a consensus problem for a system with bounded delay, and next we treat (2.1) using Lemma 2.1.

LEMma 2.4. The system (2.2) with initial condition $\phi \in \mathcal{C}\left([-\tau, 0], \mathbb{C}^{r \times 1}\right)$, solves a nontrivial consensus problem ${ }^{1}$ if and only if all the roots of

$$
\operatorname{det}\left(s I-\int_{-\tau}^{0} d \eta(\theta) e^{s \theta}\right)=0
$$

are in the open left half plane, excepting a zero root with multiplicity one, and $E_{0}=$ $\left[\begin{array}{lll}1 & \cdots & 1\end{array}\right]^{T}$ is the right null vector of

$$
\int_{-\tau}^{0} d \eta(\theta)
$$

The consensus functional $\chi: \mathcal{C}\left([-\tau, 0], \mathbb{C}^{r \times 1}\right) \rightarrow \mathbb{C}$ can be expressed as

$$
\chi(\phi)=\frac{V_{0}^{T}\left(x(\phi)(\hat{t})+\int_{-\tau}^{0} \int_{\hat{t}+\theta}^{\hat{t}} d \eta(\theta) x(\phi)(\xi) d \xi\right)}{V_{0}^{T}\left(I+\int_{-\tau}^{0} \int_{\theta}^{0} d \eta(\theta) d \xi\right) E_{0}},
$$

where $V_{0}$ is the left null vector of (2.13) and

$$
\hat{t} \geq p \tau-\limsup _{R \rightarrow \infty} \frac{\log \max _{|s|=R} \operatorname{det}\left(s I-\int_{-\tau}^{0} d \eta(\theta) e^{s \theta}\right)}{R} .
$$

Proof. The first assertion is trivial corollary of the spectrum determined growth property of the solutions of $(2.2)$, see e.g. $[10,6]$. So we restrict ourselves to the assertions on the form of the consensus functional.

Let $\mathcal{T}(t), t \geq 0$, be the time-integration operator associated with the solutions of $(2.2)$, i.e.

$$
\mathcal{T}(t) \phi=x_{t}(\phi)
$$

where $x_{t}(\phi) \in \mathcal{C}\left([-\tau, 0], \mathbb{C}^{r \times 1}\right)$ is defined by $x_{t}(\phi)(\theta)=x(\phi)(t+\theta), \theta \in[-\tau, 0]$. Note that $\mathcal{T}(t)$ is a strongly continuous semi-group. The roots of $(2.12)$, which are the eigenvalues of its infinitesimal generator $\mathcal{A}$, are infinite in number, but countable. Denote these eigenvalues by $\lambda_{i}, i \geq 0$, with $\lambda_{0}=0$, and let $P_{\lambda_{i}}$ be the spectral projection onto the corresponding generalized eigenspace $\mathcal{M}_{\lambda_{i}}$. By Theorem 8.4 of [6], one can decompose a solution $x(\phi)(t)$ on an interval $\left[t_{1}, t_{2}\right]$, where $\hat{t}<t_{1} \leq t_{2}<\infty$, in the following way:

$$
x_{t}(\phi)=\mathcal{T}(t) \phi=\sum_{i=0}^{\infty} \mathcal{T}(t) P_{\lambda_{i}} \phi .
$$

Because $\mathcal{M}_{0}=\left\{\theta \in[-\tau, 0] \mapsto c E_{0}: c \in \mathbb{C}\right\}$ we get

$$
x_{t}(\phi)=c_{0} E_{0}+\sum_{i=1}^{\infty} \mathcal{T}(t) P_{\lambda_{i}} \phi
$$

\footnotetext{
${ }^{1}$ For the system (2.2) the definition is similar to Definition 2.3, with the difference that the initial conditions should be taken from the set $\mathcal{C}\left([-\tau, 0], \mathbb{C}^{r \times 1}\right)$.
} 
where $c_{0} \in \mathbb{C}$. Since $\Re\left(\lambda_{i}\right)<0$ if $i>1$, and consequently $\lim _{t \rightarrow \infty} \mathcal{T}(t) P_{\lambda_{i}} \phi=0$, we have

$$
\chi(\phi)=c_{0} .
$$

In Section 7 of [11] it is show how the adjoint of the infinitesimal generator $\mathcal{A}$ of $\mathcal{T}(t)$ can be constructed on the space $\mathcal{C}\left([0, \tau], \mathbb{C}^{1 \times r}\right)$, starting with the bilinear form,

$$
\langle\psi, \phi\rangle=\psi(0) \phi(0)+\int_{-\tau}^{0} \int_{\theta}^{0} \psi(\xi-\theta) d \eta(\theta) \phi(\xi) d \xi
$$

where $\psi \in \mathcal{C}\left([0, \tau], \mathbb{C}^{1 \times r}\right)$ and $\phi \in \mathcal{C}\left([-\tau, 0], \mathbb{C}^{r \times 1}\right)$. By Lemma 7.3 .6 of this reference, a left eigenfunction of $\mathcal{A}$ is complementary to all right (generalized) eigenfunctions under the bilinear form (2.19), provided that they correspond to different eigenvalues. Furthermore, the left eigenfunction corresponding to the zero eigenvalue is given by the function $\theta \in[0, \tau] \mapsto V_{0}^{T}$. From these properties, the decomposition (2.17) and the fact that $\mathcal{M}_{\lambda_{i}}$ is invariant under $\mathcal{T}(t)$ we get

$$
\left\langle V_{0}^{T}, x_{t}(\phi)\right\rangle=c_{0}\left\langle V_{0}^{T}, E_{0}\right\rangle+\sum_{i=1}^{\infty}\left\langle V_{0}^{T}, T(t) P_{\lambda_{i}} \phi\right\rangle=c_{0}\left\langle V_{0}^{T}, E_{0}\right\rangle .
$$

Expressions (2.18)-(2.20) imply (2.14).

REMARK 2.5. Intuitively it is expected that the value of the consensus functional be some kind of average of the initial function over the interval $[-\tau, 0]$ and over the agents. Expression (2.14) corresponds to an average of the state at time $\hat{t}$, with $\hat{t}$ not necessarily equal to zero. To illustrate the role of the condition (2.15) we consider the system

$$
\left\{\begin{array}{l}
\dot{x}_{1}(t)=-x_{1}(t)+\frac{x_{1}(t)+x_{2}(t-\tau)}{2} \\
\dot{x}_{2}(t)=-x_{2}(t)+\frac{x_{1}(t)+x_{2}(t)}{2} .
\end{array}\right.
$$

From the characteristic equation

$$
s^{2}+s+\frac{1}{4} e^{-s \tau}=0
$$

it can be seen that it solves a consensus problem for any $\tau>0$. Because the highest power of $e^{-s \tau}$ in (2.22) is equal to one, condition (2.15) becomes $\hat{t} \geq \tau$, and the consensus functional satisfies (with $\hat{t}=\tau$ ):

$$
V(\phi)=\frac{x_{1}(\phi)(\tau)+x_{2}(\phi)(\tau)+\frac{1}{2} \int_{0}^{\tau} x_{2}(\phi)(\xi) d \xi}{2+\frac{1}{2} \tau}
$$

The underlying reason why $\hat{t}$ cannot be chosen equal to zero is that for $t \geq 0$ the solution of (2.21) with initial condition $\phi=\left[\begin{array}{ll}\phi_{1}^{T} & \phi_{2}^{T}\end{array}\right]^{T}$ is determined only by

$$
\phi_{1}(0), \quad \phi_{2}(\theta), \theta \in[-\tau, 0],
$$

while the function segment $\phi_{1}(\theta), \theta \in[-\tau, 0)$ has no influence on the future behavior and on the value of the consensus functional. By taking $\hat{t} \geq \tau$, i.e. by considering the state at time $\hat{t} \geq \tau$ which only depends on (2.23), the irrelevant part of the initial condition is ignored. 
This phenomenon is strongly related to the presence of so called small solutions, that is, solutions that vanish in a finite time (in the example the small solutions satisfy $\phi_{2}(\theta)=0, \theta \in[-\tau, 0]$ and $\left.\phi_{1}(0)=0\right)$. The condition (2.15) ensures that the contributions from such small solutions have disappeared in the solution under consideration. For more details on small solutions we refer to [6, Chapter V].

REMARK 2.6. The consensus functional proposed in Theorem 2.1 of [3] is a special case of the general functional (2.14), for compartmental time-delay systems.

Corollary 2.7. If that the system

$$
\dot{x}(t)=A_{0} x(t-\tau), \quad x(t) \in \mathbb{C}^{r \times 1},
$$

with initial condition $\phi \in \mathcal{C}\left([-\tau, 0], \mathbb{C}^{n \times 1}\right)$ solves a nontrivial consensus problem, then the consensus functional satisfies

$$
\chi(\phi)=\frac{V_{0}^{T} \phi(0)}{V_{0}^{T} E_{0}} .
$$

Proof. System (2.24) is a special case of (2.2) and can be obtained by taking

$$
\eta(\theta)=\left\{\begin{aligned}
-A_{1}, & \theta=-\tau \\
0, & \theta \in(-\tau, 0] .
\end{aligned}\right.
$$

It follows that

$$
V_{0}^{T} A_{1}=0
$$

Furthermore, integrating (2.2),

$$
\left.x_{\hat{t}}(\phi)=\phi(0)+\int_{0}^{\hat{t}} A_{1} x(\theta-\tau)\right) d \theta,
$$

makes clear that

$$
V_{0}^{T} x_{\hat{t}}(\phi)=V_{0}^{T} \phi(0) .
$$

Taking into account (2.26) and (2.27), expression (2.14) simplifies to (2.25).

Proposition 2.8. The system (2.1) solves a nontrivial consensus problem if and only if all roots of (2.3) are in the open left half plane, excepting a root at zero with multiplicity one, and $A E_{0}=0$, with $E_{0}=[1 \ldots 1]^{T}$. The corresponding consensus functional $V: \mathcal{F}\left((-\infty, 0], \mathbb{C}^{p \times 1}\right) \rightarrow \mathbb{C}$ satisfies

$$
\chi(\phi)=\frac{V_{0}^{T} \phi(0)}{V_{0}^{T} E_{0}},
$$

where $V_{0}$ is the left eigenvector of $A$ corresponding to the zero eigenvalue.

Proof. We split the proof of the first assertion in two parts:

$\Rightarrow$ We give a proof by contradiction, which allows to exclude all other possibilities. If (2.3) has a root in $\mathbb{C}^{+}$or in $j \mathbb{R} \backslash\{0\}$, then the comparison system (2.7) has a corresponding exponential solution, and, by virtue of Proposition 2.1, also (2.1). This contradicts the fact that the latter solves a consensus problem. The multiplicity of zero as a root of (2.3) is equal to its multiplicity as an eigenvalue of $A$. If $A E=0$ 
and $E \neq E_{0}$, then $x(t)=E$ is a solution of (2.1), which also leads to a contradiction. Next, we consider the case where zero is a multiple eigenvalue of $A$, yet with only one eigenvector $E_{0}$. Then there exists a generalized eigenvector $H_{0}$ such that $A H_{0}=E_{0}$. Consequently, $x(t)=H_{0}+t E_{0}$ is a solution of (2.1) (note that its restriction to $t \leq 0$ belongs to $\left.\mathcal{F}\left((-\infty, 0], \mathbb{C}^{p \times 1}\right)\right)$ and we arrive again at a contradiction. Finally, if all roots of $(2.3)$ are in $\mathbb{C}^{-}$, then the zero solution of (1.6) is asymptotically stable (Proposition 2.2), hence it does not solve a nontrivial consensus problem.

$\Leftarrow$ Following Proposition 2.1 a solution $x(\phi)(t)$ of $(2.1)$, restricted to $t \geq \tau$, also appears as component of a corresponding solution of the comparison system (2.7), which we call $z(\bar{\phi})$ in the sequel. The left and right eigenvectors of $\bar{A}+\bar{B}$ corresponding to the zero eigenvalue are given by

$$
\bar{V}_{0}=\left[\begin{array}{lll}
V_{0}^{T} & 0 \cdots 0
\end{array}\right]^{T} \text { and } \bar{E}_{0}=\left[\begin{array}{lll}
E_{0}^{T}-E_{0}^{T} & 0 \cdots 0
\end{array}\right]^{T} .
$$

Note that $\bar{V}_{0}^{T} \bar{A}=V_{0}^{T} \bar{B}=0$. Given the condition on the roots of (2.3) one proves using the same arguments as in the proof of Theorem 2.4 (based on spectral decomposition) that

$$
\lim _{t \rightarrow \infty} z(\bar{\phi})(t)=\frac{\bar{V}_{0}^{T}\left(z_{\hat{t}}(\bar{\phi})(0)+\bar{B} \int_{\hat{t}-\tau}^{\hat{t}} z(\phi)(\theta) d \theta\right)}{\bar{V}_{0}^{T}(I+\tau \bar{B}) \bar{E}_{0}} \bar{E}_{0}=\frac{V_{0}^{T} \phi(0)}{V_{0}^{T} E_{0}} \bar{E}_{0},
$$

where

$$
\hat{t} \geq p(n+1) \tau-\limsup _{R \rightarrow \infty} \frac{\log F(R)}{R}, \quad F(R)=\max _{|s|=R} \operatorname{det}\left(s I-\bar{A}-\bar{B} e^{-s \tau}\right) .
$$

It follows that

$$
\lim _{t \rightarrow \infty} x(\phi)(t)=\frac{V_{0}^{T} \phi(0)}{V_{0}^{T} E_{0}} E_{0},
$$

implying that (2.7) solves a consensus problem.

The assertion on the consensus function follows from (2.30).

REMARK 2.9. Expressions (2.25) and (2.28) also follow from a simple geometric argument. As in both cases $V_{0}^{T} \dot{x}(t)=0$ a solution $x(\phi)(t)$ is constrained to the plane $V_{0}^{T} x=V_{0}^{T} \phi(0)$ for all $t \geq 0$. Furthermore, a constant stationary solution must be a multiple of $E_{0}$. Thus $x^{*}(\phi)=\lim _{t \rightarrow \infty} x(\phi)(t)$ satisfies the equations

$$
\left\{\begin{array}{l}
V_{0}^{T} x^{*}(\phi)=V_{0}^{T} \phi(0) \\
x^{*}(\phi)=\chi(\phi) E_{0}
\end{array}\right.
$$

which can be interpreted as the intersection of the plane through $\phi(0)$ and perpendicular to $V_{0}$ with a line with slope $E_{0}$. A similar argument was used in Section $X$ of [19].

3. Conditions for the realization of a consensus. We perform a stability analysis of the system (1.6)-(1.7) in the $(T, \tau)$ parameter space. In particular we give necessary and sufficient conditions such that it solves a consensus problem.

The system (1.6)-(1.7) can be written in the form (2.1), yet has some special properties due to the induced structure of $A$, which we outline first. Next, we make an analysis of an auxiliary scalar equation and, finally, we present the main results. 
3.1. Properties. The system (1.6)-(1.7) is of the form (2.1), where $A=\left[a_{k, l}\right]$ is defined as

$$
a_{k, l}=\left\{\begin{array}{cc}
\alpha_{k, l}, & k \neq l, \\
-\sum_{i=1, i \neq k}^{p} \alpha_{k, i}, & k=l
\end{array}\right.
$$

Note that in the context of multi-agent systems $-A$ is typically called the graph Laplacian of (1.1). By construction $A$ has the following property:

Property 3.1. All eigenvalues of $A$, defined by (3.1), are in $\mathbb{C}^{-}$, excepting a zero eigenvalue with multiplicity one.

Proof. $A$ is a Metzler matrix with zero row sums. Furthermore, the graph (1.1) is strongly connected. Under these conditions the statement of the proposition follows from Theorem 1 and Theorem 2 of [19].

Note that zero also appears as a root of (2.3) and (3.1), whatever the values of $T$, $\tau$ and $n$. If all other roots are in $\mathbb{C}^{-}$, we have from Proposition 2.8 that the system (1.6)-(1.7) solves a (nontrivial) consensus problem with delay. In the car following application the consensus variables are the speed of the vehicles. This means that, whatever the initial values, the speed of the vehicles will eventually converge to a common value (which depends on the initial values).

In the sequel we shall use the following terminology to characterize parameter values in the $(T, \tau)$ space for which a consensus is reached:

DEFINITION 3.2. The consensus region of (1.6)-(1.7) in the $(T, \tau)$ parameter space is the set of parameters $(T, \tau)$ for which the system (1.6)-(1.7) solves a consensus problem.

3.2. Analysis of an auxiliary function. Motivated by Property 3.1 and the factorization of the characteristic equation (2.3) as (2.4) we are led to the study the zeros of the function

$$
\xi(s ; T, \tau):=s(1+s T)^{n} \mathrm{e}^{s \tau}-\mu, \quad \mu \in \mathbb{C}^{-},
$$

as a function of the parameters $T$ and $\tau$. Note that the zeros of (3.2) are in $\mathbb{C}^{-}$if and only if the roots of

$$
s-\frac{\mu e^{-s \tau}}{(1+s T)^{n}}=0
$$

are in $\mathbb{C}^{-}$. We need the following lemmas:

LEMMA 3.3. In the parameter domain a change of the number of zeros $\xi$ in the closed right half plane is invariably associated with zeros crossing the imaginary axis.

Proof. Follows from the continuous dependence of the individual zeros with respect to the parameters and the fact that zeros of $\xi$ in the closed right half plane satisfy

$$
|s| \leq|\mu| \frac{\left|e^{-s \tau}\right|}{|1+s T|^{n}} \leq|\mu|
$$

which excludes roots coming from the point at infinity. $\square$

LEMMA 3.4. If the function (3.2) has a zero on the imaginary axis, then the multiplicity of this zero is equal to one. Furthermore, an increase of $\tau$ leads to a crossing towards $\mathbb{C}^{+}$. If $\tau=0$, then also an increase of $T$ leads to a crossing towards $\mathbb{C}^{+}$. 
Proof. The first assertion is due to the fact that

$$
\frac{\partial \xi}{\partial s}(j \omega ; T, \tau)=(1+j \omega T)^{n-1} e^{j \omega \tau}(1+j \omega T+j \omega n+j \omega \tau(1+j \omega T))
$$

is nonzero for all $\omega \in \mathbb{R}$.

Next, let $j \bar{\omega}, \bar{\omega} \in \mathbb{R}$, be an isolated zero of $(3.2)$ for $(T, \tau)=(\bar{T}, \bar{\tau})$. It is clear that $\bar{\omega} \neq 0$. Since the zeros of (3.2) behave continuously w.r.t. the parameters $T$ and $\tau$, there exists a function

$$
r: \mathbb{R}^{+} \times \mathbb{R}^{+} \rightarrow \mathbb{R}, \quad(T, \tau) \mapsto r(T, \tau),
$$

satisfying $r(\bar{T}, \bar{\tau})=j \bar{\omega}$ and

$$
\xi(r(T, \tau) ; T, \tau)=0
$$

Differentiating $(3.3)$ at $(\bar{T}, \bar{\tau})$ yields

$$
\frac{\partial r}{\partial \tau}(\bar{T}, \bar{\tau})=-\frac{\frac{\partial \xi}{\partial \tau}(j \bar{\omega} ; \bar{T}, \bar{\tau})}{\frac{\partial \xi}{\partial s}(j \bar{\omega} ; \bar{T}, \bar{\tau})}, \quad \frac{\partial r}{\partial T}(\bar{T}, \bar{\tau})=-\frac{\frac{\partial \xi}{\partial T}(j \bar{\omega} ; \bar{T}, \bar{\tau})}{\frac{\partial \xi}{\partial s}(j \bar{\omega} ; \bar{T}, \bar{\tau})},
$$

from which one obtains:

$$
\Re\left(\left(\frac{\partial r}{\partial \tau}(\bar{T}, \bar{\tau})\right)^{-1}\right)=\frac{1}{\bar{\omega}^{2}}+\frac{n \bar{T}}{1+\bar{\omega}^{2} \bar{T}^{2}}, \quad \Re\left(\left(\frac{\partial r}{\partial T}(\bar{T}, \bar{\tau})\right)^{-1}\right)=\frac{1}{\bar{\omega}^{2}}-\frac{\bar{T} \bar{\tau}}{n} .
$$

The first expression is strictly positive, the second if $\bar{\tau}=0$. This implies the second assertion. $\square$

We adopt a two-stage approach, similar to the one proposed by [17]. First we characterize the zeros distribution of $(3.2)$ as a function of $T$, under the condition $\tau=0$ :

Proposition 3.5. If $\mu$ is real and $n=1$, then the zeros of $\xi(s ; T, 0)$ are in $\mathbb{C}^{-}$ for all $T \geq 0$. Otherwise, the zeros of $\xi(s ; T, 0)$ are in $\mathbb{C}^{-}$if and only if $T \in\left[0, T_{\mu}\right)$, where

$$
T_{\mu}=\frac{\tan \left(\frac{|\angle(\mu)|-\frac{\pi}{2}}{n}\right)}{|\mu|\left[\cos \left(\frac{|\angle(\mu)|-\frac{\pi}{2}}{n}\right)\right]^{n}} .
$$

Proof. Assume that $\xi(s ; T, 0)$ has a zero on the imaginary axis for some value of $T$. Then there exists a corresponding frequency $\omega>0$ such that either

$$
\xi(j \omega / T ; T, 0)=0 \text { or } \xi(-j \omega / T ; T, 0)=0,
$$

which is equivalent to

$$
T=\frac{ \pm j \omega(1 \pm j \omega)^{n}}{\mu}
$$

If $n=1$ and $\mu$ is real, then the right hand size of (3.5) cannot be real, whatever the value of $\omega$. Hence, $\xi(s ; T, 0)$ cannot have zeros on the imaginary axis. Combining this fact with the continuity of the zeros w.r.t. $T$ and the assumption $\mu \in \mathbb{C}^{-}$, i.e. all zeros are in $\mathbb{C}^{-}$for $T=0$, yields the first statement of the proposition. 
If $n>1$ or $\Im(\mu) \neq 0$, then there always exist pairs $(T, \omega), T>0$, which satisfy (3.5), that is, $\xi(s ; T, 0)$ has zero $+j \omega / T$ or $-j \omega / T$. Because by Lemma 3.4 the corresponding crossing direction of the imaginary axis is towards $\mathbb{C}^{+}$as $T$ is increased, and because $\mu \in \mathbb{C}^{-}$, all zeros of $\xi(s ; T, 0)$ are in $\mathbb{C}^{-}$if and only if $T \in\left[0, T_{\mu}\right)$, where

$$
T_{\mu}=\min \{T>0:(T, \omega) \text { satisfies (3.5) for some } \omega>0\} .
$$

Since the functions $\omega>0 \rightarrow\left| \pm j \omega(1 \pm j \omega)^{n}\right| /|\mu|$ are strictly increasing, $T_{\mu}$ is determined by the first intersection of one of the two curves

$$
\omega>0 \rightarrow \frac{ \pm j \omega(1 \pm j \omega)^{n}}{\mu}
$$

with the positive real axis, as $\omega$ is increased from zero. In what follows we distinguish between two cases:

Case 1: $\Im(\mu) \geq 0$. The first intersection of (3.6) with the positive real axis is due to the curve corresponding the plus sign. It is characterized by $\omega=\bar{\omega}$, satisfying

$$
\angle(j \bar{\omega})+\angle\left((1+j \bar{\omega})^{n}\right)-\angle(\mu)=0 .
$$

It follows that

$$
\bar{\omega}=\tan (\angle(1+j \bar{\omega}))=\tan \left(\frac{\angle(\mu)-\pi / 2}{n}\right)
$$

and

$$
(1+j \bar{\omega})^{n}=\left(1+\bar{\omega}^{2}\right)^{n / 2} e^{j(\angle \mu-\pi / 2)}=\frac{e^{j(\angle(\mu)-\pi / 2)}}{\left[\cos \left(\frac{\angle(\mu)-\pi / 2}{n}\right)\right]^{n}} .
$$

Expression (3.4) is obtained when substituting (3.7) and (3.8) in

$$
T_{\mu}=\frac{j \bar{\omega}(1+j \bar{\omega})^{n}}{\mu} .
$$

Case 2: $\Im(\mu)<0$. The first intersection of (3.6) with the positive real axis is due to the curve corresponding the minus sign, and characterized by $\omega=\hat{\omega}$, where

$$
\angle(-j \hat{\omega})+\angle\left((1-j \hat{\omega})^{n}\right)+|\angle(\mu)|=0 .
$$

One can proceed as in the former case.

Second, we fix $T$ and characterize the zeros distribution of (3.2) as a function of the delay parameter $\tau$. We make use of the functions $g_{n}$ described by Definition 1.1.

Proposition 3.6. The function $\xi(s ; T, \tau)$, with $T$ fixed, has a zero on the imaginary axis for some delay value $\tau$ if and only if $\tau \in \mathcal{T}_{\mu}(T)$, where ${ }^{2}$

$$
\begin{aligned}
\mathcal{T}_{\mu}(T)=\left\{\tau \geq 0 \mid \tau=\frac{ \pm \angle(\mu)-\angle\left(j \omega(1+j \omega)^{n}\right)+2 \pi l}{\omega / T},\right. & \\
l & \left.=\mathbb{Z}, \omega=g_{n}(T|\mu|)\right\} .
\end{aligned}
$$

\footnotetext{
${ }^{2}$ The right hand side of expression (3.9) is not defined for $T=0$, which implies $\omega=g_{n}(T|\mu|)=0$. In that case one should interpret $g_{n}(T|\mu|) / T$ as $\lim _{T \rightarrow 0+} g_{n}(T|\mu|) / T=|\mu|$ and $\angle\left(j \omega(1+j \omega)^{n}\right)$ as $\lim _{\omega \rightarrow 0+} \angle\left(j \omega(1+j \omega)^{n}\right)=\pi / 2$.
} 
Furthermore, all zeros are in $\mathbb{C}^{-}$if and only if the zeros of $\xi(s ; T, 0)$ are in $\mathbb{C}^{-}$and $\tau \in\left[0, \tau_{\mu}(T)\right)$, where

$$
\tau_{\mu}(T)=\frac{|\angle(\mu)|-\angle\left(j \omega(1+j \omega)^{n}\right)}{\omega / T}, \quad \omega=g_{n}(T|\mu|) .
$$

Proof. The function $\xi(s ; T, \tau)$ has a zero $j \omega / T$ or $-j \omega / T, \omega>0$, if and only if

$$
j \omega(1+j \omega)^{n}=T \mu e^{-j \omega \tau / T}, \text { or }-\mathrm{\jmath} \omega(1-j \omega)^{n}=T \mu e^{j \omega \tau / T} .
$$

Equating modulus and phase of left and righthand side leads to (3.9).

From the second assertion of Lemma 3.4 it follows that $\xi(s ; T, \tau)$ has its zeros in the open left half plane if and only if $\xi(s ; T, 0)$ does so and $\tau \in\left[0, \tau_{m}(T)\right)$, where

$$
\tau_{m}(T)=\min _{\tau>0} \mathcal{T}_{\mu}(T)
$$

It remains to prove that $\tau_{m}(T)=\tau_{\mu}(T)$, with $\tau_{\mu}(T)$ defined by (3.10), for all $T$ such that $\xi(s ; T, 0)$ has its zeros in $\mathbb{C}^{-}$(described by Proposition 3.5$)$.

For sufficiently small $T$, and thus sufficiently small $\omega(T)=g_{n}(T|\mu|)$, we have

$$
0<|\angle(\mu)|-\angle\left(j \omega(T)(1+j \omega(T))^{n}\right) \leq \pi / 2
$$

and

$$
\begin{aligned}
& -|\angle(\mu)|-\angle\left(j \omega(T)(1+j \omega(T))^{n}\right)+2 \pi \\
& \quad=|\angle(\mu)|-\angle\left(j \omega(T)(1+j \omega(T))^{n}\right)+2(\pi-|\angle(\mu)|) \\
& \quad \geq|\angle(\mu)|-\angle\left(j \omega(T)(1+j \omega(T))^{n}\right),
\end{aligned}
$$

which imply

$$
\tau_{m}(T)=\tau_{\mu}(T)
$$

As the function $T \rightarrow \omega(T)$ is monotonically increasing, (3.11)-(3.12), and consequently (3.13), either hold for all $T \geq 0$, or for values of $T$ which belong to a finite number of intervals. In the latter case one of these intervals is given by $\left[0, T_{m}\right)$, with $T_{m}$ satisfying

$$
|\angle(\mu)|-\angle\left(j \omega\left(T_{m}\right)\left(1+j \omega\left(T_{m}\right)\right)^{n}\right)=0 .
$$

It follows that $0 \in \mathcal{T}_{\mu}\left(T_{m}\right)$, which implies on its turn that $T_{\mu}$ exists and $T_{m} \geq T_{\mu}$. We conclude $\tau_{\mu}(T)=\tau_{m}(T)$ for $T \in\left[0, T_{\mu}\right)$.

REMARK 3.7. In expression (3.9), the plus sign of $\pm \angle \mu$ refers to zeros on the positive imaginary axis, the minus sign to zeros on the negative imaginary axis. If $\Im(\mu) \neq 0$, the corresponding values of $\tau$ are in general different. Finally, combining Proposition 3.5 and Proposition 3.6 yields:

Proposition 3.8. If $\mu$ is real and $n=1$, then the zeros of (3.2) are in $\mathbb{C}^{-}$if and only if $T \in[0, \infty)$ and $\tau \in\left[0, \tau_{\mu}(T)\right)$. Otherwise, the zeros are in $\mathbb{C}^{-}$if and only if $T \in\left[0, T_{\mu}\right)$ and $\tau \in\left[0, \tau_{\mu}(T)\right)$. 
3.3. Main results. Taking into account the factorization of (2.3) as (2.4), Property 3.1 and Proposition 3.8, we obtain the following characterization of the consensus region (cf. Definition 3.2) of the system (1.6)-(1.7) in the $(T, \tau)$ space:

THEOREM 3.9. If $n=1$ and all eigenvalues of $A$, defined by (3.1), are real, then the consensus region of (1.6)-(1.7) in the $(T, \tau)$ plane is unbounded and characterized by

$$
T \in[0, \infty), \quad \tau \in\left[0, \tau^{*}(T)\right),
$$

where

$$
\tau^{*}(T)=\min _{k=1, \ldots, p, \mu_{k} \neq 0} \frac{\left|\angle\left(\mu_{k}\right)\right|-\angle\left(j \omega_{k}(T)\left(1+j \omega_{k}(T)\right)^{n}\right)}{\frac{\omega_{k}(T)}{T}}
$$

and $\omega_{k}(T)=g_{n}\left(T\left|\mu_{k}\right|\right)$.

Otherwise, the consensus region is bounded and characterized by

$$
T \in\left[0, T^{*}\right), \quad \tau \in\left[0, \tau^{*}(T)\right),
$$

where

$$
T^{*}=\min _{k=1, \ldots, p, \Im\left(\mu_{k}\right)>0} \frac{\tan \left(\frac{\angle\left(\mu_{k}\right)-\frac{\pi}{2}}{n}\right)}{\left|\mu_{k}\right|\left[\cos \left(\frac{\angle\left(\mu_{k}\right)-\frac{\pi}{2}}{n}\right)\right]^{n}}
$$

and $\tau^{*}(T)$ is given by (3.14).

Based on this result the consensus region of (1.6)-(1.7) can be computed fully automatically. For large $p$ the overall computational complexity is determined by the computation of the eigenvalues of the $p$-by- $p$ matrix $A$.

Theorem 3.9 does not make assumptions on the multiplicity of the eigenvalues of $A$ and is generally applicable. If $A$ has eigenvalues with multiplicity larger than one, then the stability study of (1.6)-(1.7) is even facilitated as not all factors in (2.4) are different. The following proposition clarifies the connection between multiple eigenvalues of $A$ and multiple eigenvalues of the comparison system (2.7) of (1.6)(1.7):

Proposition 3.10. Let $\hat{\mu}$ be a nonzero eigenvalue of $A$ with multiplicity $m_{1}$ and a corresponding eigenspace of dimension $m_{2}$. Then the roots of

$$
s(1+s T)^{n} e^{s \tau}-\hat{\mu}=0
$$

with multiplicity $m_{3}$ are eigenvalues of the comparison system (2.7) with multiplicity $m_{1} m_{3}$ and an eigenspace of dimension $m_{2}$. Furthermore, if $m_{3}=1$, then these roots smoothly depend on the parameters $T$ and $\tau$.

Proof. Let $\hat{s}$ be a zero of (3.16) with multiplicity $m_{3}$. The factorization (2.4) implies that $\hat{s}$ is an eigenvalue of (2.7) with multiplicity $m_{1} m_{3}$. Following [10], the corresponding eigenfunctions have the form $e^{\hat{s} \theta} Z, \theta \in\left([-\tau, 0]\right.$, where $Z \in \mathbb{R}^{p(n+1) \times 1}$ satisfies

$$
\left(\hat{s} I-\bar{A}-\bar{B} e^{-\hat{s} \tau}\right) Z=0 .
$$


With $Z$ partitioned according to the structure of $\bar{A}$ and $\bar{B}$ as $Z=\left[\begin{array}{lll}X^{T} & Y_{0}^{T} \cdots Y_{n-1}^{T}\end{array}\right]^{T}$, writing out (3.17) yields

$$
\left(\hat{s} I-A \frac{e^{-\hat{s} \tau}}{(1+\hat{s} T)^{n}}\right) X=0, \quad Y_{i}=\frac{\hat{s}^{i}}{(1+\hat{s} T)^{n}} X, \quad i=0, \ldots, n-1 .
$$

Since

$$
\left(\hat{s} I-A \frac{e^{-\hat{s} \tau}}{(1+\hat{s} T)^{n}}\right) X=\frac{e^{-\hat{s} \tau}}{(1+\hat{s} T)^{n}}(\hat{\mu} I-A) X,
$$

$X$ must be an eigenvector of $A$ corresponding $\hat{\mu}$. Hence, the dimension of the eigenspace of $\hat{s}$ is equal to $m_{2}$.

If $m_{3}=1$, then $\hat{s}$ is an isolated root of (3.16), and the last statement can be proven using the arguments spelled out in the proof of Lemma 3.4.

REMARK 3.11. If $m_{1}>m_{2}$, then the roots of (3.16) with multiplicity one (this is for instance always the case for roots on the imaginary axis, see Proposition 3.5) are multiple, non-semisimple eigenvalues of (2.7), yet they smoothly depend on the parameters $T$ and $\tau$. Small changes of $T$ and $\tau$ do not lead to a splitting of these multiple eigenvalues.

The next proposition addresses a scaling property of the consensus region:

Proposition 3.12. If the matrix $A$ is scaled with a factor $\epsilon>0$, then the consensus region of (1.6)-(1.7) in the $(T, \tau)$ plane is scaled with a factor $\epsilon^{-1}$ in both directions.

Proof. Follows from the scaling property

$$
\operatorname{det}\left(s I-\epsilon A \frac{e^{-s \tau}}{(1+T s)^{n}}\right)=\epsilon^{p} \operatorname{det}\left(\bar{s} I-A \frac{e^{-\bar{s}(\epsilon \tau)}}{(1+\overline{(}(\epsilon T) \bar{s})^{n}}\right), \quad \bar{s}=s / \epsilon .
$$

REMARK 3.13. Proposition 3.12 implies an inherent trade-off between the rate with which the undelayed system $(\tau=T=0)$ reaches a consensus (determined by the rightmost nonzero eigenvalue of $A$ ), and the robustness of this stability property w.r.t. delays. Such an observation was already made in [19], where the case of a symmetric matrix $A$ and a point-wise delay was dealt with.

In the remainder of this section, we refine Theorem 3.9 to two special case where exploiting the additional structure leads to a simpler characterization of the consensus region, and also allows an analytical expression for the solutions corresponding to an onset of instability. The proof of the resulting propositions can be found in the appendix.

The following result corresponds to the situation where all cars/drivers have an identical behavior and the reaction of a driver is determined by the preceding car only:

Proposition 3.14. Consider the system (1.6)-(1.7), where

$$
\alpha_{k, l}= \begin{cases}\alpha>0, & \lfloor k-l\rfloor=1 \\ 0, & \text { otherwise. }\end{cases}
$$

If $n=1$, and $p=2$ then the consensus region in the $(T, \tau)$ plane is unbounded and characterized by

$$
T \in[0, \infty), \quad \tau \in\left[0, \tau^{*}(T)\right),
$$


where

$$
\tau^{*}(T)=\frac{\frac{\pi}{p}-n \arctan (\omega(T))}{\frac{\omega(T)}{T}}, \quad \omega(T)=g_{n}\left(2 \alpha T \sin \left(\frac{\pi}{p}\right)\right) .
$$

Otherwise, the consensus region is bounded and characterized by

$$
T \in\left[0, T^{*}\right), \quad \tau \in\left[0, \tau^{*}(T)\right),
$$

where

$$
T^{*}=\frac{\tan \left(\frac{\pi}{p n}\right)}{2 \alpha \sin \left(\frac{\pi}{p}\right)\left(\cos \left(\frac{\pi}{p n}\right)\right)^{n}}
$$

and $\tau^{*}(T)$ is given by (3.19).

For $\tau=\tau^{*}(T)$ the stationary solutions are backwards traveling waves:

$$
\left[\begin{array}{c}
v_{1}^{s}(t) \\
v_{2}^{s}(t) \\
\vdots \\
v_{p}^{s}(t)
\end{array}\right]=C_{1}\left[\begin{array}{c}
\cos \left(\frac{\omega(T)}{T} t+\varphi\right) \\
\cos \left(\frac{\omega(T)}{T} t+\varphi-\frac{2 \pi}{p}\right) \\
\vdots \\
\cos \left(\frac{\omega(T)}{T} t+\varphi-\frac{2 \pi(p-1)}{p}\right)
\end{array}\right]+C_{2}\left[\begin{array}{c}
1 \\
1 \\
\vdots \\
1
\end{array}\right],
$$

where $\omega(T)$ is defined in (3.19) and the constants $C_{1}, C_{2}$ and $\phi$ depend on the initial conditions.

Second, we consider the case where (1.6)-(1.7) is of the form (2.1), with the matrix A symmetric. Although this is not a realistic assumption from the car following application point of view, it makes sense in the context of consensus algorithms for multi-agent systems. The symmetry of $A$ there corresponds to an undirected network topology.

Proposition 3.15. Consider the system (1.6)-(1.7) with A symmetric. If $n=1$, then the consensus region of (1.6)-(1.7) in the $(T, \tau)$ plane is unbounded and characterized by

$$
T \in[0, \infty), \quad \tau \in\left[0, \tau^{*}(T)\right),
$$

where

$$
\tau^{*}(T)=\frac{\frac{\pi}{2}-n \arctan (\omega(T))}{\frac{\omega(T)}{T}}, \quad \omega(T)=g_{n}\left(T\left|\lambda_{\max }(A)\right|\right) .
$$

Otherwise, the consensus region is bounded and characterized by

$$
T \in\left[0, T^{*}\right), \quad \tau \in\left[0, \tau^{*}(T)\right),
$$

where

$$
T^{*}=\frac{\tan \left(\frac{\pi}{2 n}\right)}{\left|\lambda_{\max }(A)\right|\left[\cos \left(\frac{\pi}{2 n}\right)\right]^{n}}
$$

and $\tau^{*}(T)$ is given by (3.22). 
If, in addition,

$$
\alpha_{k, l}= \begin{cases}\alpha>0, & \lfloor k-l\rfloor=1 \text { or }\lfloor l-k\rfloor=1 \\ 0, & \text { otherwise }\end{cases}
$$

then

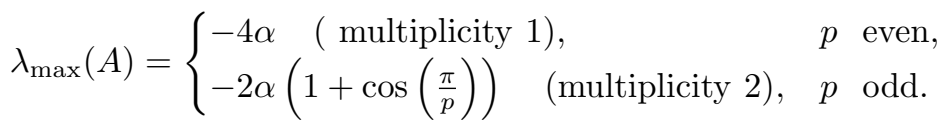

The stationary solutions for $\tau=\tau^{*}(T)$ take the form:

$$
\left[\begin{array}{c}
v_{1}^{s}(t) \\
\vdots \\
v_{p-1}^{s}(t) \\
v_{p}^{s}(t)
\end{array}\right]=C_{1}\left[\begin{array}{c}
(-1)^{p-1} \\
\vdots \\
(-1) \\
1
\end{array}\right] \cos \left(\frac{\omega(T)}{T} t+\varphi_{1}\right)+C_{2}\left[\begin{array}{c}
1 \\
\vdots \\
1 \\
1
\end{array}\right]
$$

if $p$ is even, and

$$
\begin{aligned}
& {\left[\begin{array}{c}
v_{1}^{s}(t) \\
\vdots \\
v_{p-1}^{s}(t) \\
v_{p}^{s}(t)
\end{array}\right] }=C_{3}\left[\begin{array}{c}
(-1)^{p-1} \cos \left(\frac{\pi(p-1)}{p}\right) \\
\vdots \\
(-1) \cos \left(\frac{\pi .1}{p}\right) \\
1
\end{array}\right] \cos \left(\frac{\omega(T)}{T} t+\varphi_{2}\right) \\
&+C_{4}\left[\begin{array}{c}
(-1)^{p-1} \sin \left(\frac{\pi(p-1)}{p}\right) \\
\vdots \\
(-1) \sin \left(\frac{\pi .1}{p}\right) \\
0
\end{array}\right] \cos \left(\frac{\omega(T)}{T} t+\varphi_{3}\right)+C_{5}\left[\begin{array}{c}
1 \\
\vdots \\
1 \\
1
\end{array}\right]
\end{aligned}
$$

if $p$ is odd. The constants $C_{1}, \ldots, C_{5}$ and $\varphi_{1}, \ldots, \varphi_{3}$ depend on the initial conditions.

REMARK 3.16. Under the assumption of the above proposition the consensus functional satisfies

$$
V(\phi)=\frac{1}{p}[1 \cdots 1] \phi(0)
$$

This follows from (2.8), taken into account that $V_{0}=E_{0}$ if $A$ is symmetric. Hence, under the conditions of the above proposition an average consensus problem is solved, in the sense that all components of a solution $x(\phi)(t)$ converge to the average of these components at the starting time, i.e. $\phi(0)$. Observe that $\phi(\theta), \theta<0$, has no influence on the limit reached.

REMARK 3.17. Expression (3.23) reduces to the statement of Theorem 10 in [19] if $T \rightarrow 0+$.

Let us briefly compare the stationary solutions (3.21) with (3.25)-(3.26). In the former case, the directed 'network topology' (a driver only reacts -with some delayon its predecessor, and not the other way around) naturally leads to a backwards 


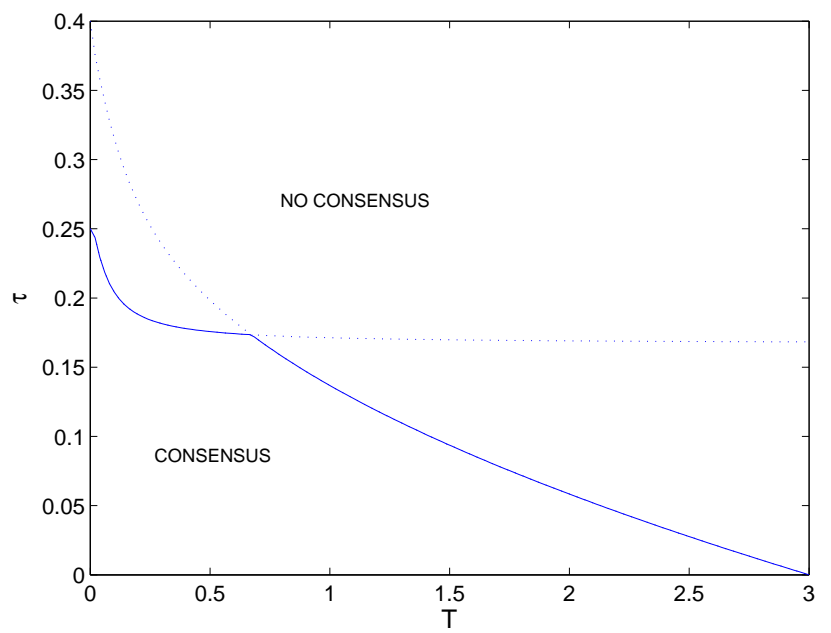

FIG. 4.1. Boundary of the consensus regions of (1.6)-(1.7) with parameters (4.1) (solid curve). Boundaries of stability regions of (4.2) (dotted curves).

traveling wave. In the latter case, one would from the symmetry of the coupling intuitively expect a stationary wave, where subsequent agents oscillate in anti-phase. This is indeed the case for (3.25) which holds if $p$ is even. However, if $p$ is odd, such a solution is incompatible with the ring configuration, and (3.26) holds. If $p$ is large, (3.26) can be seen as an approximation of an stationary wave with subsequent agents oscillating in anti-phase that is compatible with the ring configuration.

4. Examples. As a first example we compute the consensus regions in the $(T, \tau)$ plane of system (1.6)-(1.7) with $n=1$ and

$$
A=\left[\begin{array}{rrrr}
-5 & 0 & 0 & 5 \\
1 & -1 & 0 & 0 \\
0 & 1 & -1 & 0 \\
0 & 0 & 5 & -5
\end{array}\right]
$$

The eigenvalues of this matrix are given by

$$
\mu_{1}=-6, \mu_{2}=\bar{\mu}_{3}=-3+j, \mu_{4}=0 .
$$

An application of Theorem 3.9 yields the consensus region

$$
T \in[0,3), \tau \in\left[0, \tau^{*}(T)\right),
$$

where the function $T \rightarrow \tau^{*}(T)$ is displayed in Figure 4.1 as a solid line. The dotted lines bound the 'stability' regions of the auxiliary equations

$$
s(1+s T) e^{s \tau}-\mu_{1,2}=0,
$$

which are described by Proposition 3.8. The stability region corresponding $\mu_{1}$ is unbounded as $\mu_{1}$ is real and $n=1$.

To illustrate the asymptotic behavior when the number of cars is large, we take a system satisfying condition (3.18) of Proposition 3.14. Figure 4.2 shows the consensus 


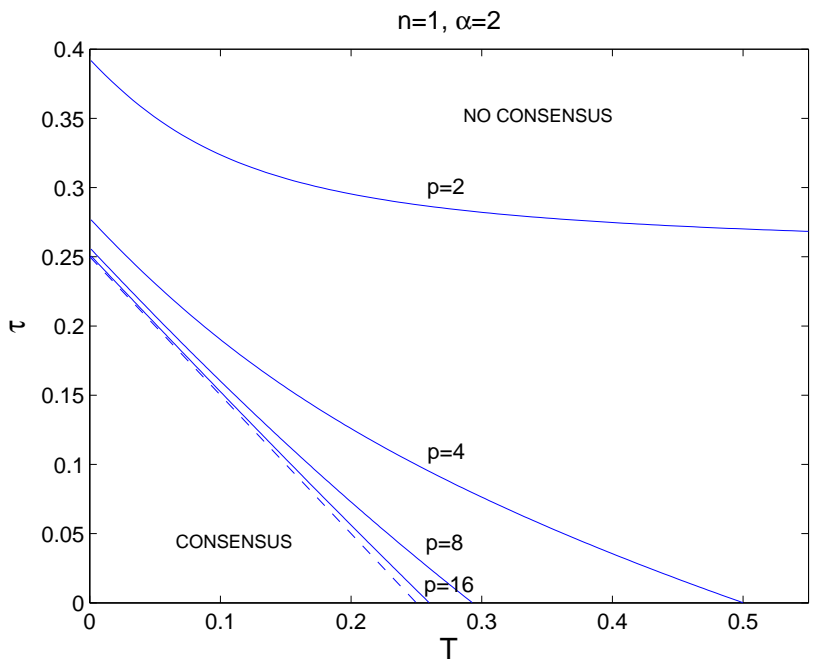

FIG. 4.2. Boundary of the consensus region of a system satisfying (3.18), with parameters $\alpha=2$ and $n=1$.

region in the $(T, \tau)$ plane for $\alpha=2, n=1$ and $p=2^{k}, k=1, \ldots, 4$. It it follows from (3.19) that as $p \rightarrow \infty$, the boundary of the consensus region uniformly converges to the function

$$
\tau_{l}^{*}(T)=\frac{1}{2 \alpha}-n T
$$

indicated in Figure 4.2 with a dashed line.

Finally, we consider the system

$$
\dot{v}_{k}(t)=\sum_{l=1}^{3} \alpha_{k,\lfloor k-l\rfloor} \int_{0}^{\infty} f(\theta)\left(v_{\lfloor k-l\rfloor}(t-\theta)-v_{k}(t-\theta)\right) \mathrm{d} \theta, \quad k=1, \ldots, 1000,
$$

where $f$ is given by (1.7), with $n=2$. The parameters

$$
\begin{aligned}
& \alpha_{k,\lfloor k-1\rfloor} \in[1,5], \\
& \alpha_{k,\lfloor k-2\rfloor} \in\left[0, \frac{3}{4} \alpha_{k,\lfloor k-1\rfloor}\right], \\
& \alpha_{k,\lfloor k-3\rfloor} \in\left[0, \frac{3}{4} \alpha_{k,\lfloor k-2\rfloor}\right], \quad k=1, \ldots, 1000
\end{aligned}
$$

are randomly generated according to a uniform distribution over the above intervals. For 30 sets of parameters obtained in this way, the consensus region in the $(T, \tau)$ plane was computed. The results are displayed in Figure 4.3. 


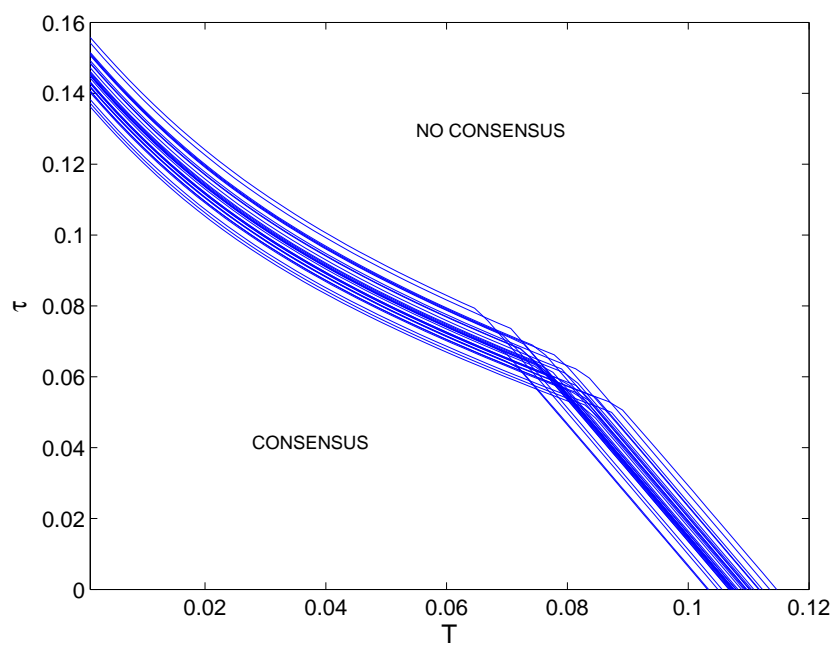

Fig. 4.3. Consensus region of (4.3)-(4.4), for 30 different data sets.

5. Other models. For general time-delay systems of retarded type with multiple constant delays and distributed delays with gamma-distribution kernels, stability and/or consensus regions of equilibria in a two-parameter spaces can be computed semi-automatically by numerical continuation, see for instance [14] and the package DDE-BIFTOOL [7]. Such an approach involves the discretization of an infinitedimensional evolutionary operator, associated with the time-delay system, to compute the rightmost eigenvalues. Roughly speaking the computation of the boundary of a stability or consensus region involves solving $r$ eigenvalue problems of dimension $p q \times p q$, where $p$ is the dimension of the system, $q$ denotes the number of discretization points, and $r$ is the number of points on the stability crossing curves where stability information is checked. When using Theorem 3.9 only one eigenvalue problem of dimension $p \times p$ needs to be solved to determine the complete stability region of (1.6)-(1.7) in the $(T, \tau)$ space. The underlying reason is that the structure of the system allowed a decomposition into small sub-problems, which is apparent from the form of the characteristic equation (2.4).

Let us now take a brief look at the so-called optimal velocity models, also frequency encountered in the literature. The linearization around the equilibrium of the models studied in [1], respectively [5, 21, 22] and the references therein, takes the form

$$
\tau_{k} \ddot{x}_{k}(t)+\dot{x}_{k}(t-\tau)=\alpha\left(x_{\lfloor k-1\rfloor}(t-\tau)-x_{k}(t-\tau)\right), k=1, \ldots, p,
$$

respectively

$$
\tau_{k} \ddot{x}_{k}(t)+\dot{x}_{k}(t)=\alpha\left(x_{\lfloor k-1\rfloor}(t-\tau)-x_{k}(t-\tau)\right), k=1, \ldots, p .
$$

In both cases, $x_{k}$ denotes the position of the $k-t h$ vehicle. The left hand side models the dynamics of the vehicle and the right hand side is the reference velocity, which is a function of the distance to the preceding vehicle and models the behavior of the 
driver. Note that (5.1) and (5.2) can be generalized to:

$$
\begin{aligned}
\ddot{x}_{k}(t)=\int_{0}^{\infty} f(\theta)\left(\left(\sum_{i=1}^{p-1} \alpha_{k,\lfloor k-i\rfloor}\left(x_{\lfloor k-i\rfloor}(t-\theta)-x_{k}(t-\theta)\right)\right)\right. \\
\left.\quad-\beta_{k} \dot{x}_{k}(t-\theta)\right) \mathrm{d} \theta-\gamma_{k} \dot{x}_{k}(t), k=1, \ldots, p,
\end{aligned}
$$

with $f$ given by (1.7). General purpose tools for the stability and bifurcation analysis of time-delay systems like DDE-BIFTOOL can be applied directly to (5.3). However, if all vehicles have similar characteristics (but not necessarily the drivers), that is, $\beta_{k} \equiv \beta, \gamma_{k} \equiv \gamma$, then the characteristic equation can again be factorized:

$$
\begin{aligned}
0 & =\operatorname{det}\left(s(s+\gamma) I-(A-\beta s I) \frac{e^{-s \tau}}{(1+s T)^{n}}\right) \\
& =\Pi_{k=1}^{p}\left(s(s+\gamma)-\frac{\left(\mu_{k}-\beta s\right) e^{-s \tau}}{(1+s T)^{n}}\right)
\end{aligned}
$$

where $A$ is given by $(3.1)$ and $\mu_{1}, \ldots \mu_{p}$ denote its eigenvalues. Also here, its beneficial to exploit this decomposition into small sub-problems, in particular if the number of vehicles is large.

6. Conclusions. The stability analysis of a linear system including a $\gamma$-distributed delay with a gap for modeling traffic flow dynamics was considered. A complete characterization of the regions in the delay-parameter space, where a consensus is reached for all initial conditions, was obtained. In particular, by exploiting the structure of the system analytical expressions were derived for the bounds on the parameters of the delay distribution. These expressions give rise to a fully automatic computation of the consensus region, whose complexity is determined by the computation of the eigenvalues of one matrix with dimensions equal to the number of vehicles. Some illustrative examples were presented.

From a theoretical point of view some stability theory for linear systems with $\gamma$ distributed delays was developed. As this type of distributed delays is characterized by kernels with an infinite support, which prohibits a full spectral decomposition of the solutions, the relation between the growth properties of the solutions and the roots of an appropriate characteristic equations was established via a comparison system with constant delays. Necessary and sufficient conditions for the realization of a consensus problem and an explicit construction of the consensus functional were provided, for both systems with constant and distributed delays.

7. Acknowledgements. The authors wish to thank the editors and the reviewers for their careful reading of the paper and their constructive comments.

This article presents results of the Research Project IAP P5, funded by the programme on Interuniversity Poles of Attraction, initiated by the Belgian State, Prime Minister's Office for Science, Technology and Culture and of OPTEC, the Optimization in Engineering Center of the K.U.Leuven. The work of W. MichiELS and S.-I. NiCulescu was partially funded by PAI Tournesol Bilateral Project (France, Flanders): "Distributed delays in dynamical systems: Analysis and applications" (20062007). The work of C.-I. MORĂRESCU was (partially) supported through a European Community Marie Curie Fellowship and in the framework of the CTS, contract number: HPMT-CT-2001-00278. W. MichiELs is a postdoctoral fellow of the Fund for Scientific Research -Flanders (Belgium). 
[1] Bando, M., Hasebe, K., Nakanishi, K. and Nakayama, A.: Analysis of optimal veloicity model with explicit delay. Phys. Rev. E, 58 (1998) 5429-5435.

[2] Chandler, R.E., Herman, R. and Montroll, E. W.: Traffic Dynamics: Analysis of Stability in Car Following, Operational Research, $\mathbf{7}(1)$ (1958) 165-184.

[3] Chellaboina, V., Haddad, W.M., Hui, Q. and Ramakrishnan, J.: On systems state equipartitioning and semistability in network dynamical systems with arbitrary time-delays. in Proc. 45th IEEE Conf. Dec. Contr., San Diego, CL (2007) 3461-3466.

[4] Chen, J.: Static output feedback stabilization for SISO systems and related problems: solutions via generalized eigenvalues. Control - Theory and Advanced Tech., 10 (1995) 2233-2244.

[5] Davis, L.C.: Modifications of the optimal velocity traffic model to include delay due to driver reaction time. Physica A, 319 (2003) 557-567.

[6] Diekmann, O., van Gils, S.A., Verduyn Lunel, S.M. and Walther, H.-O.: Delay equations. Functional-, Complex-, and Nonlinear Analysis (Applied Mathematical Sciences, vol. 110, Springer-Verlag: New York, 1995).

[7] Engelborghs, K. Luzyanina, T. and Samaey, G.:DDE-BIFTOOL v. 2.00: a Matlab package for bifurcation analysis of delay differential equations. Technical report TW-330, Department of Computer Science, K.U.Leuven, Leuven, Belgium, 2001

[8] Green, M.: How long does it take to stop? in Transportation Human Factors, 2 (2000) 195-216.

[9] Gu, K., Kharitonov, V.L. and Chen, J.: Stability of time-delay systems (Birkhauser: Boston, 2003).

[10] Hale, J.K. and Verduyn Lunel, S.M.: Introduction to functional differential equations (Applied Mathematical Sciences, vol. 99, Springer-Verlag: New York, 1993).

[11] Hale, J.: Theory of functional differential equations (Applied Mathematical Sciences, vol. 3, Springer-Verlag: New York, 1977).

[12] Helbing, D.: Traffic and Related Self-Driven Many-Particle Systems, Reviews of Modern Physics, 73 (2001) 1067-1141.

[13] Jadbabaie, A., Lin, J. and Morse, A.S.: Coordination of groups of mobile autonomous agents using nearest neighbor rules. IEEE Transactions on Automatic Control, 48(6) (2003) 9881001.

[14] Luzyanina, T. and Roose, D.: Equations with distributed delays: bifurcation analysis using computational tools for discrete delay equations. Functional Differential Equations, 11(12) (2004) 87-92.

[15] Michiels, W. and Niculescu, S.-I.: Stability and stabilization of time-delay systems. An eigenvalue based approch (Advances in Design and Control, vol. 12, SIAM, 2007).

[16] Morărescu, C.I.: Qualitative analysis of distributed delay systems. Methodology and algorithms (Ph.D. thesis, University of Bucharest, Romania/Université de Technologie de Compiègne, France, September 2006).

[17] Morărescu, C.I., Niculescu, S.-I. and Michiels, W.: Asymptotic stability of some distributed delay systems: An algebraic approach. International Journal of Tomography and Statistics, 7(F07) (2007) 128-132.

[18] Niculescu, S.-I. and Abdallah, C.T.: Delay effects on static output feedback stabilization. in Proc. 39th IEEE Conf. Dec. Contr., Sydney (Australia) December 2000.

[19] Olfati-Saber, R. and Murray, R.M.: Consensus problems in networks of agents with switching topology and time-delays. IEEE Transactions on Automatic Control, 49(9) (2004) 15201533.

[20] Olfati-Saber, R., Fax, A. and Murray, R.M.: Consensus and cooperation in networked multiagent systems. in Proc. IEEE 95 (2007) 215-233.

[21] Orosz, G., Krauskopf, B. and Wilson, R.E.: Bifurcations and multiple traffic jams in a carfollowing model with reaction-time delay. Physica D, 211(3-4) (2005) 277-293.

[22] Orosz, G. and Stépán, G.: Subcritical Hopf bifurcations in a car-following model with reactiontime delay. Proc. Royal Society A, 462(2073) (2006) 2643-2670.

[23] Ren, W., Beard, R.W. and Atkins, E.: Information consensus in multivehicle cooperative control. IEEE Control Systems Magazine, 27(2) (2007)71-82.

[24] Rothery, R.W.: Transportation Research Board (TRB) Special Report 165, in Traffic Flow Theory, 2nd Edition, N. H. Gartner, C. J. Messner, and A. J. Rathi, Eds., (1998).

[25] Sipahi, R. and Niculescu S.-I.: Analytical stability study of a deterministic car following model under multiple delay interactions. in Proc. 6th IFAC Workshop on Time Delay Systems, L'Aquila (Italy) July 2006.

[26] Sipahi, R. and Niculescu S.-I.: Some remarks on the characterization of delay interactions in deterministic car following models. in Proc. 17th Symp. MTNS, Kyoto (Japan) July 2006.

[27] Sipahi, R., Atay, F.M., and Niculescu S.-I.: On stability analysis of some traffic flow models with distributed memory effect of the drivers. SIAM J. Applied Mathematics, 68(3) (2007) 
738-759.

Appendix A. Proof of Proposition 3.14. The system matrix A, defined by (3.1), becomes circulant under condition (3.18). It is readily verified that its eigenvalues are given by

$$
\mu_{k}=-\alpha+\alpha e^{j \frac{2 \pi}{p} k}, \quad k=1, \ldots p
$$

with corresponding eigenvectors

$$
\left[\left(1+\mu_{k} / \alpha\right)^{p-1} \cdots\left(1+\mu_{k} / \alpha\right)^{2}\left(1+\mu_{k} / \alpha\right) 1\right]^{T} .
$$

The eigenvalues of $A$ are real if and only if $p=2$.

Let $q=p / 2$ if $p$ is even and $q=(p-1) / 2$ otherwise. ¿From (A.1) it follows that

$$
\begin{aligned}
& \left|\mu_{k}\right|=2 \alpha \sin \left(\frac{\pi}{p} k\right), \\
& \angle\left(\mu_{k}\right)=\frac{\pi}{2}+\frac{\pi}{p} k, \quad k=1, \ldots, q .
\end{aligned}
$$

Substituting these values in (3.14) yields

$$
\tau^{*}(T)=\min _{k=1, \ldots, q} \frac{\left(\frac{\pi}{p} k-n \arctan \left(\omega_{k}(T)\right)\right) T}{\omega_{k}(T)},
$$

where

$$
\left|j \omega_{k}(T)\left(1+j \omega_{k}(T)\right)^{n}\right|=2 \alpha T \sin \left(\frac{\pi}{p} k\right), \quad k=1, \ldots, q
$$

We have

$$
\frac{k}{\omega_{k}(T)}=\frac{k\left|\left(1+j \omega_{k}(T)\right)^{n}\right|}{2 \alpha T \sin \left(\frac{\pi}{p} k\right)} \geq \frac{\left|\left(1+j \omega_{1}(T)\right)^{n}\right|}{2 \alpha T \sin \left(\frac{\pi}{p}\right)}=\frac{1}{\omega_{1}(T)}, \quad k=1, \ldots, q,
$$

where we used $\omega_{k}(T) \geq \omega_{1}(T)$ and $\sin (k x) \leq k \sin (x)$ for $x \in[0, \pi /(2 k)]$. This estimate and the fact that the function $x \rightarrow \arctan (x) / x$ is decreasing on $[0, \infty)$ lead to

$$
\begin{aligned}
\frac{\left(\frac{\pi}{p} k-n \arctan \left(\omega_{k}(T)\right)\right) T}{\omega_{k}(T)} & =\left(\frac{\pi k}{p \omega_{k}(T)}-\frac{n \arctan \left(\omega_{k}(T)\right)}{\omega_{k}(T)}\right) T \\
& >\left(\frac{\pi}{p \omega_{1}(T)}-\frac{n \arctan \left(\omega_{1}(T)\right)}{\omega_{1}(T)}\right) T \\
& =\frac{\left(\frac{\pi}{p}-n \arctan \left(\omega_{1}(T)\right)\right) T}{\omega_{1}(T)}, \quad k=1, \ldots, q .
\end{aligned}
$$

From (A.4) and (A.5), one obtains (3.19).

Using (A.3) expression (3.15) becomes

$$
T^{*}=\min _{k=1, \ldots, q} \frac{\tan \left(\frac{\pi}{p n} k\right)}{2 \alpha \sin \left(\frac{\pi}{p} k\right)\left(\cos \left(\frac{\pi}{p n} k\right)\right)^{n}} .
$$

Taking into account that the function $x \mapsto \frac{\tan (x / n)}{\sin (x) \cos ^{n}(x / n)}$ is increasing on $[0, \pi / 2]$, one obtains (3.20). 
Finally we consider the stationary solutions for $\tau=\tau^{*}(T)$. ¿From the proof of Proposition 3.6, (A.1), (A.3)-(A.5) it follows that the equation

$$
s(1+s T)^{n} e^{s \tau^{*}(T)} \mp \mu_{1}=0
$$

has solutions $s^{ \pm}= \pm \frac{j \omega}{T}$ where $\omega=g_{n}\left(2 \alpha T \sin \left(\frac{\pi}{p}\right)\right)$. From Lemma 3.4, (A.1) and Proposition 3.10 these solutions are eigenvalues of (2.7) with multiplicity one. As spelled out in the proof of Proposition 3.10 the corresponding eigenfunctions have the form $Z^{ \pm} e^{\frac{ \pm j \omega T}{T}}$, where $Z^{ \pm}$can be partitioned as $Z=\left[X^{ \pm T} Y_{0}^{ \pm T} \ldots Y_{n-1}^{ \pm T}\right]^{T}$, with

$$
e^{\frac{ \pm j \omega t}{T}} X^{ \pm}=e^{\frac{ \pm j \omega t}{T}}\left[e^{ \pm j \frac{2 \pi(p-1)}{p}} \cdots e^{ \pm j \frac{2 \pi}{p}} 1\right]^{T}
$$

In the above we used (A.2). Note from Proposition 2.1 that (A.6) is a solution of $(2.1)$.

The solution (3.21) is a linear combination of (A.6), the solutions corresponding to the eigenvalues $\pm j \omega / T$, and $[1 \cdots 1]^{T}$, the eigenfunction corresponding to the zero eigenvalue.

Appendix B. Proof of Proposition 3.15. The expressions (3.22) and (3.23) follow from directly from Theorem 3.9, when taking into account that the eigenvalues of $A$ are negative real.

Under the condition (3.24) the eigenvalues of $A$ are given by

$$
\mu_{k}=-2 \alpha+2 \alpha \cos \left(\frac{2 \pi}{p}(k-1)\right), k=1, \ldots, q,
$$

where $q=(p+2) / 2$ is $p$ is even and $q=(p+1) / 2$ if $p$ is odd. All eigenvalues have multiplicity two, excepting $\mu_{1}$ and, if $p$ is even, $\mu_{\frac{p+2}{2}}$. The corresponding eigenvectors are

$$
\left[\begin{array}{c}
\cos \left(\frac{2 \pi(k-1) \cdot(p-1)}{p}\right) \\
\vdots \\
\cos \left(\frac{2 \pi(k-1) \cdot 1}{p}\right) \\
1
\end{array}\right] \text { and }\left[\begin{array}{c}
\sin \left(\frac{2 \pi(k-1) \cdot(p-1)}{p}\right) \\
\vdots \\
\sin \left(\frac{2 \pi(k-1) \cdot 1}{p}\right) \\
0
\end{array}\right]
$$

One can proceed as in the proof of Proposition 3.14. 